\title{
SARS-CoV-2 induced coagulopathy and potential role of anticoagulation: Scoping review of literature
}

\author{
Zehra Naseem ${ }^{1}$, Muhammad Mussab Khakwani' ${ }^{1}$ Maaha Ayub ${ }^{1}$, Ahmed Ayaz ${ }^{1}$, Bushra Jamil ${ }^{2}$, \\ Ainan Arshad ${ }^{2}$ \\ ${ }^{1}$ Medical College, Aga Khan University, Karachi; ${ }^{2}$ Department of Medicine, Aga Khan University, Karachi, Pakistan
}

\begin{abstract}
Severe acute respiratory syndrome coronavirus 2 (SARS$\mathrm{CoV}-2$ ) can vary on a spectrum of asymptomatic disease to rarer
\end{abstract}

Correspondence: Ainan Arshad, Senior Instructor and Hospitalist, Associate Program Director, Internal Medicine Residency Program, Department of Medicine, Faculty office building, 2nd floor, Aga Khan University, National Stadium Road, Karachi 74800, Pakistan.

E-mail: ainan_arshad@hotmail.com

Key words: SARSCoV-2; COVID-19; hypercoagulability; coagulopathy; anticoagulation.

Contributions: ZN, MMK, MA, literature search, original draft writing; AA, AAr, BJ, study concepts, editing, final draft and overall supervision. All the authors have read and approved the final version of the manuscript and agreed to be accountable for all aspects of the work.

Conflict of interest: The authors declare that they have no competing interests, and all authors confirm accuracy.

Funding: This research did not receive any specific grant from funding agencies in the public, commercial, or not-for-profit sectors.

Ethics approval and consent to participate: Not applicable.

Consent for publication: Not applicable.

Availability of data and materials: Data sharing is not applicable to this article as no datasets were generated or analyzed during the current study.

Received for publication: 31 May 2021.

Accepted for publication: 5 October 2021.

Publisher's note: All claims expressed in this article are solely those of the authors and do not necessarily represent those of their affiliated organizations, or those of the publisher, the editors and the reviewers. Any product that may be evaluated in this article or claim that may be made by its manufacturer is not guaranteed or endorsed by the publisher.

${ }^{\circ}$ Copyright: the Author(s), 2022

Licensee PAGEPress, Italy

Monaldi Archives for Chest Disease 2022; 92:1958

doi: 10.4081/monaldi.2022.1958

This article is distributed under the terms of the Creative Commons Attribution-NonCommercial International License (CC BY-NC 4.0) which permits any noncommercial use, distribution, and reproduction in any medium, provided the original author(s) and source are credited. manifestations like hypercoagulability especially among elderly patients admitted in the intensive care unit and those with preexisting comorbidities. The exact mechanism behind this phenomenon is still unclear, however studies have shown an association with elevated cytokines and severe inflammatory response which encompasses this disease. Hypercoagulability can be limited to the lungs, or present as systemic manifestations of arterial and venous thrombosis leading to mortal outcomes. Thus, careful evaluation of risk factors should be performed by physicians and treatment with anticoagulants should be modified accordingly. All coronavirus disease 2019 (COVID-19) in-patients should receive thromboprophylactic therapy, with increased dosages administered to patients with increased disease severity or those with a high risk. D-dimer levels and sepsis-induced coagulopathy score aid in identifying high risk patients and predicting outcome. This article highlights the pathophysiology behind hypercoagulability, its clinical associations and discusses therapeutic modalities to combat this fatal consequence of SARS-CoV-2.

\section{Introduction}

Severe acute respiratory syndrome coronavirus 2 (SARSCoV-2) first originated as a pneumonia-causing organism in the Hubei province of China, and is responsible for causing coronavirus disease 2019 (COVID-19) [1]. By March 2020, it had affected thousands of people and caused 300,000 fatalities [2], rendering it a pandemic. SARS-CoV-2 is transmitted from one infected person to another predominantly via respiratory droplets and close human contact. COVID-19 may vary from mild to severe infection, depending on the immunocompetence level of the patient. Although it primarily manifests as fever, cough and fatigue, a variety of other symptoms such as gastrointestinal problems, dyspnea and in severe cases, sepsis may also occur [3].

SARS-CoV-2, similar to other coronaviruses, is known to cause coagulopathy which occurs as a result of thrombosis in blood vessels. This may take place in a number of different organs including lungs, heart, liver and brain [4]. Coagulopathy as a result of SARS-CoV-2 infection has a poor prognosis and may occur in spite of anticoagulation therapy. Although the exact mechanism involved in causing coagulopathy in COVID-19 is still unclear, a number of different hypotheses have been put forward [5]. This review aims to identify the pathophysiology of hypercoagulability in SARS-CoV-2 infection, along with its various pathologies and provides a comprehensive review of the anticoagulation therapies which can be used in different patient populations in order to tackle this cause of morbidity and mortality among COVID-19 patients. 


\section{Pathophysiology of coagulopathy}

When a virus enters a host, inflammation and the coagulation system is activated in order to combat pathogens [6]. Previous literature has associated SARS-CoV-2 with changes in coagulation profile which is correlated with a worse outcome [7,8]. Though still debated, the mechanism behind this phenomenon is likely due to high levels of inflammatory mediators causing direct damage to blood vessel walls resulting in platelet aggregation and exposure of tissue factor causing coagulation [9], along with vessel thrombosis and angiopathy [10]. Moreover, anticoagulation proteins like plasmin are inhibited due to decreased activation of plasminogen and increased inhibition of plasminogen activator [11]. However, in pathologies where plasmin activity is already high such as hypertension and diabetes, there is increased fibrinolysis and high levels of D-dimer with increased pathogenicity of SARS-CoV-2 due to splitting of its surface proteins [12]. Previous data has also shown a correlation between inflammatory mediators like IL-6 and development of venous thromboembolism (VTE) [13].

Another suggested theory is activation of angiotensin converting enzyme 2 (ACE 2) receptor on vascular endothelial cells by coronavirus $\mathrm{S}$ protein which leads to increased angiotensin 2 levels with a further increase in tissue factor. This leads to endothelial dysfunction and thrombosis [14]. This results in high C1 levels which inhibits conversion of plasminogen to plasmin, thus shifting the balance from fibrinolysis to thrombosis. Additionally, angiotensin 2 increases smooth muscle contraction of pulmonary vasculature along with pneumonia induced hypoxic conditions, resulting in blood stasis and a prothrombotic state $[15,16]$. Moreover, the vascular endothelial cells can be damaged directly by SARS-CoV-2 [10].

There have also been rare findings of production of antiphospholipid antibodies which play a role in thrombosis [17]. Meanwhile, those with congenital bleeding disorders have a relatively low incidence of worsening disease and death rates due to the hypercoagulative nature of COVID-19 [18]. Furthermore, lack of significant thrombocytopenia demonstrates that the hypercoagulability in COVID-19 is not identical to the disseminated intravascular coagulation (DIC) caused by other infections [19].

\section{Laboratory biomarkers}

Among patients who are admitted to in-patient settings, manifestations of hypercoagulability such as VTE and pulmonary embolism (PE) occur often [20], along with high levels of markers of hypercoagulability like fibrinogen and D-dimer [21]. Assessment of hypercoagulation involves measurement of various parameters, one of them being D-dimer levels. The prevalence of high levels of D-dimer is $46.4 \%$ among COVID-19 patients, with $43 \%$ being in less serious cases and $60 \%$ in severe cases [22]. Patients presenting with respiratory compromise due to acute respiratory distress syndrome (ARDS) have D-dimer levels of $5 \mathrm{mg} / \mathrm{L}$ (ten times higher than upper cut off), high fibrinogen and fibrin degradation products [23], while thromboelastometry has shown the formation of stable thrombi [24]. Moreover, levels of PT, aPTT and von Willebrand factor (vWF) are also elevated with low levels of antithrombin 3 [25]. These findings show that sepsis-induced coagulopathy (SIC) is an occurrence in SARS-CoV-2, where $71.4 \%$ of the mortal cases satisfied its criteria as compared to $0.6 \%$ of the non-mortal cases [25].
Hypercoagulability and its associated pathologies carry a poor prognosis among individuals with COVID-19 [15], such that high levels of D-dimer ( $>1 \mu \mathrm{g} / \mathrm{ml})$ are associated with an increased risk of mortality in 28 days [25]. Studies on autopsy findings among fatal cases of COVID-19 have shown VTE to be a prevalent cause of death in these cases [26]. In view of hypercoagulation and associated mortality risk, it is advised to give high doses of anticoagulants in cases where there are high serum D-dimer levels ( $>3 \mathrm{mg} / \mathrm{l})$ $[27,28]$. However, D-dimer is not used as a diagnostic marker due to less specificity [20]. It is also debated whether non-hospitalized patients should be treated with anticoagulants or anti platelet drugs. Thus, health care professionals should work according to the recommendations of most recent data from reliable sources [29].

Previous studies, such as the one by Mortus et al. show that $90 \%$ of their ICU patients with SARS-CoV-2 infection had an elevated thrombotic parameter (high fibrinogen) while $62 \%$ of the patients had manifestations of thrombosis including arterial, venous, dialysis and filter catheter thrombosis [2]. In addition to this, thrombosis in portal vein, splenic infarcts and stroke can also result [30]. As a consequence, this hypercoagulative state can lead to cardiac and vascular pathologies like myocardial infarction (MI) and eventually death [31].

\section{Clinical associations}

\section{Venous thromboembolism}

SARS-CoV-2 can result in VTE, as suggested by elevated nonspecific D-dimer levels in 68\% of admitted COVID-19 patients [32], and some case reports [33]. According to a study by Klot et al., the incidence of VTE among ICU patients was $48 \%$ at 2 weeks [34]. In another study, the prevalence of VTE among ICU patients despite anticoagulant prophylactic measures was 5-15\% [35], while Cui et al had $25 \%$ of their patients with VTE [36]. In addition to this, Klol et al. reported VTE incidence of $27 \%$ and $3.7 \%$ arterial pathologies even though prophylaxis with Low Molecular Weight Heparin (LMWH) was administered [31]. In another study among SARS-CoV-1 cases, DVT and VTE was present in $20.5 \%$ of the cases [37], while Criel et al. had 7.3\% of their cases with VTE, with most of them being ICU patients [35].

VTE can occur in the abdominal organs, pelvic organs or the lower limbs [30]. SARS-CoV-2 can occasionally lead to the development of sepsis, associated with high levels of inflammatory mediators like IL-6, IL-8 and TNF-alpha [38], neutrophil extracellular traps (NETs) and polyphosphates [39], along with a disruption of the anti-coagulation nature of endothelial cells [39]. This leads to a stimulation of the coagulation cascade resulting in microvascular thrombosis, VTE and DIC [40, 41]. Moreover, microthrombi, high LDH and ferritin, mildly high PT and aPTT all indicate pathology of blood vessels with associated thrombosis [6].

Studies have shown a higher incidence of VTE among the elderly, stemming from changes in the immune system which alter the presentation of mRNA of T cells leading to VTE [42]. Along with this, the presence of comorbidities such as hypertension and diabetes which are associated with high expression of ACE-2 receptor (the receptor for viral entry) [14] also lead to increased VTE among patients.

PE in COVID-19 may result from the high levels of inflammatory cytokines leading to endothelial damage, poor oxygenation and DIC, in spite of medical prophylaxis for deep venous thrombosis (DVT) [10]. PE can even present after acute inflammatory stage, raising concerns for a persistent hypercoagulable state [43]. 
PE leads to micro thrombi which affect proximal and segmental arteries in the lungs with the development of right ventricular damage in some patients [20]. PE is associated with higher levels of Ddimer as compared to patients without PE, whereas low platelets and an elevated PT are correlated with a poorer prognosis [32]. Bompard and his colleagues' study showed an incidence of PE of $24 \%$, where $30-70 \%$ of the cases were from ICU patients and $12-$ $27 \%$ from other in-patients [20]. On the other hand, a study among SARS-CoV-1 patients showed $11.4 \%$ of the patients to have PE [37]. Cases have also been reported where deterioration of respiratory function due to bilateral PE led to hypoxia [10], which further required mechanical ventilation and re-hospitalizations [20]. Respiratory failure in PE occurs due to microthrombi and fibrin in the alveoli and pulmonary interstitium, as the terminal steps in the coagulation system [16]. Thus, deteriorating respiratory function warrants suspected thrombosis, with the use of thromboelastometry as a possible screening method and prophylactic anticoagulants/mechanical ventilation as treatment/prevention [16].

Where specific tests for PE diagnosis are unavailable, physicians can benefit from symptom recognition of PE such as hemoptysis (present in $13 \%$ of PE patients) and right ventricular pressure overload signs through EKG or computed tomography angiography (CTA) [23]. It may also be beneficial to measure serum levels of D-dimer, fibrinogen, platelets and development of DIC in order to detect PE earlier [32]. Additionally, prophylactic measures for VTE such as LMWH administration should be done for severe cases, irrespective of D-dimer levels as these levels are not helpful in distinguishing thrombus formation from high levels due to serious illness [40].

\section{Arterial thrombosis and its cardiac and neurologic sequalae}

The hypercoagulative state can also result in thrombosis in arteries of large or medium caliber, as was reported by a retrospective analysis in $6.1 \%$ of SARS-CoV-2 cases [30], including $3.7 \%$ of ICU patients [31]. A retrospective cohort study reported cases of arterial thrombosis presenting as MI [32], while a case series has demonstrated the presence of ST segment elevation among COVID-19 patients where all of the cases had high serum levels of D-dimer [44]. According to Shi et al, 19.7\% of their patients had elevated troponin I levels, demonstrating a probable MI. Those with MI had more death rates $(51.2 \%)$ as compared to those without MI, (4.5\%) with high troponin I levels being positively associated with fatality [45]. Troponin $\mathrm{T}$ levels were found to be positively correlated with levels of white blood cells, inflammatory markers like C-reactive protein, procalcitonin and markers of ventricular failure like $\mathrm{N}$-terminal pro B type natriuretic peptide, creatine kinase and myoglobin [46]. In addition to this, elderly patients with comorbid conditions of hypertension and diabetes were more likely to have cardiac complications, which in turn translates to a higher risk of ARDS and death [45]. Pathogenesis underlying this finding is high myocardial oxygen demand, hypoxia, rupture of atherosclerotic plaques due to high levels of cytokines, endothelial dysfunction of coronary arteries and thrombi in coronary vasculature [47]. Moreover, SARS-CoV-2 can enter vessel endothelium and cardiac muscle directly via ACE-2 receptor and cause direct damage, which can affect individuals without prior cardiovascular compromise, although this finding is not very common [45]. High incidence of cardiac damage is seen among those individuals with prior cardiovascular compromise due to elevated myocardial stress from inflammation and high cytokine load [48].

Neurological sequelae of hypercoagulability in SARS-CoV-2 include ischemic stroke, as was present in $2.8 \%$ of patients with a prevalence of $5.7 \%$ among critical cases due to occlusion of large caliber arteries [49]. According to a Spanish registry, the most common neurological finding in COVID-19 is confusion $(28.3 \%)$ followed by ischemic stroke (22.8\%) [50]. A study by Zhang et al showed that 2 of their patients with ischemic stroke had high Ddimer and fibrinogen levels along with the presence of antiphospholipid antibodies [17]. Proposed etiologies for ischemic stroke include a hypercoagulable state, microangiopathy, dysfunction of endothelial cells, emboli from cardiac origin due to MI or alteration of blood flow mechanisms due to cardiogenic shock or sepsis [51]. Despite serious consequences of ischemic stroke, premature treatment with anticoagulants is debated due to the probability of development of hemorrhage [52].

\section{Microcirculation dysfunction, microthrombi in organs and organ failure}

In addition to the role of microthrombi in VTE, they also play a role in the pathogenesis of organ failure in COVID-19 [53]. Meta analysis studies such as the one by Cao et al have shown the presence of organ dysfunction in $8.5 \%$ of the cases [54]. Inflammatory mediators including TNF-alpha, IL-1beta, IL-6, IFN-gamma, monocyte chemotactin protein-1, interferon-inducible protein 10 , macrophage inflammatory protein 1 alpha and G-CSF play a role in the pathogenesis of microthrombi and coagulation [40,55]. According to postmortem findings, microthrombi, occlusion of blood vessels and increased thickness of vessels have been observed in the lungs and extrapulmonary organs [56]. Additionally, complement proteins like $\mathrm{C} 4 \mathrm{~d}$, membrane attack complex and mannose binding lectin associated serine protease 2 have been isolated from the lungs and the vessels of the skin, hinting at the role of complement system in vessel thrombosis [57]. Inflammatory cells were also present in these vessels with additional findings of apoptosis due to isolation of caspase 3 activity [10]. Moreover, Mehta et al. compared the findings in COVID-19 with hemophagocytic lymphohistiocytosis (HLH) and demonstrated that the two diseases share some similarities such as high levels of ferritin and LDH, ARDS and organ dysfunction [54].

\section{SARS-CoV-2 hypercoagulability, pregnancy and cancer}

With pre-existing changes in the coagulation profile during pregnancy in favor of a pro-coagulative state, high fibrinogen levels in SARS-CoV-2 infection correlate with a high likelihood of thrombosis among pregnant females [40]. Thus, in order to decrease thrombosis related complications, ISTH has recommended the administration of LMWH as a prophylactic measure in pregnant, SARS-CoV-2 infected patients if they have the following features: high D-dimer, high PT, platelets $<100 \times 10^{9} / 1$ or fibrinogen $<2$ g/l [58]. Similar to this, cancer patients with SARS-CoV-2 are susceptible to thrombosis and its sequelae, thus prophylaxis with anticoagulants is recommended [59].

\section{Role of anticoagulation}

Role of anticoagulation in treating COVID-19 patients is becoming increasingly moot due to limited data. Considering the risk and occurrence of coagulopathy in COVID-19 patients, it is imperative to find out the potential role of anticoagulation in these patients.

A retrospective study including 449 people in China showed that generally there was no significant difference in 28-day mortality between patients receiving LMWH and those not receiving LMWH. However the use of LMWH reduced 28 day mortality by 
$24.2 \%$ in extremely sick patients with six times the normal Ddimer levels and an SIC score of more than 4\% [28]. Hence, it can be deduced that anticoagulation therapy is effective in patients with markedly elevated D-dimer levels and a high SIC score. Increased D-dimer concentrations of more than double the upper limit of normal is considered a good predictive biomarker for developing VTE and demands for a subsequent need for anticoagulation therapy [60].

In a retrospective series of 2773 SARS-CoV-2 patients, $28 \%$ received anticoagulation therapy. As a whole, it had negligible impact on mortality rate. In-hospital survival was $78 \%$ for patients receiving anticoagulation while it was $77 \%$ for those who did not. However, in intubated patients, anticoagulation therapy had a strong association with in-hospital survival (71\% versus 37\%) [61].

In another publication by Ranucci et al., 16 SARS-CoV-2 patients in the ICU were given high doses of Clopidogrel and Nadroparin and subsequently, no VTE event was reported among these patients. On the downside the study had a small sample size and there was no control group [24]. Therefore, it can be concluded that existing evidence shows that anticoagulation therapy has a part to play in preventing and resolving SARS-CoV-2 induced coagulopathy among severe cases.

\section{VTE/PE prophylaxis and treatment}

Considering the high risk of VTE in COVID-19 patients, VTE prophylaxis is recommended in the management of all in-hospital patients unless the risk of bleeding outweighs the risk of thrombosis. The Swiss Society of Hematology however, offered dose adjustments according to the patient's body weight and D-dimer levels [62]. For non-hospitalized SARS-CoV-2 patients, anticoagulation therapy should not be initiated unless there is some indication [63].

Multiple studies have shown that there is still increased risk of VTE in critically ill SARS-CoV-2 infected patients despite receiving prophylactic anticoagulation doses $[34,60,64]$. Therefore, it is being suggested that escalated anticoagulation dose should be given to ICU patients to prevent VTE but has not yet been proven to be effective. A clinical trial, yet to take place, would be ideal to determine the appropriate approach in such cases.

Post-discharge VTE prophylaxis is uncommon and is not recommended due to risk of bleeding. However, in low bleed risk patients with the following conditions, it may be worthwhile to continue post-discharge VTE prophylaxis:

- If the patients were admitted to the ICU, intubated, sedated, and paralyzed for multiple days

- If the patients have VTE risk factors at the time of discharge (e.g., diminished mobility, profound weakness, not at baseline physical status) [65].
Nonetheless, currently there is no direct scientific evidence to address post-discharge VTE prophylaxis in SARS-CoV-2 infected patients.

\section{Drugs of choice}

LMWH is the anticoagulant drug of choice for SARS-CoV-2 patients whereas unfractioned Heparin can be used as an alternative in patients with marked renal impairment i.e., creatinine clearance of less than $30 \mathrm{ml} / \mathrm{min}$ [66]. Initially, elevated levels of D-dimer and fibrinogen degradation products decreased significantly after treatment with LMWH, indicating an improvement in the hypercoagulable state in SARS-CoV-2 infected patients. It can be administered subcutaneously once or twice daily and does not require frequent monitoring to ensure that it is effectively dosed [65]. Apart from its role in anticoagulation, Heparin also has anti-inflammatory properties. It down-regulates IL-6 which is a pleiotropic pro-inflammatory cytokine released in response to SARS-CoV-2 [67]. In the setting of heparin-induced thrombocytopenia, fondaparinux is recommended [63]. A few studies have suggested that combination therapy of heparin and nafamostat may be able to establish improved control over SARS-CoV2 induced coagulopathy [62]. Nafamostat is a serine protease inhibitor that potentiates the anticoagulant effect of Heparin in patients. Table 1 illustrates the recommended therapies for different patients. More literature is required to establish the indication of using this combination in battling SARS-CoV-2 induced coagulopathy.

It is advised that oral anticoagulants, including warfarin, dabigatran, and factor Xa inhibitors betrixaban, apixaban and rivaroxaban should not be prescribed to patients receiving anti-retroviral medications due to possible interactions between the two [65]. Similarly, Testa et al. recommended switching to parenteral Heparin from oral anticoagulants in COVID-19 patients, considering the possible drug-drug interactions and variability in vitamin $\mathrm{K}$ metabolism, leading to unstable PT/INR and possibly liver impairment and heart failure in patients treated with vitamin $\mathrm{K}$ anticoagulants [68].

At our institute Aga Khan University Hospital in Karachi, Enoxaparin (LMWH) is the drug of choice for SARS-CoV-2 induced coagulopathy. Other drugs regularly given to these patients include dexamethasone, immunosuppressant tocilizumab and antiviral remdesivir. As of now, heparin has not been reported to interact with the above medications used in the management of SARS-CoV-2.

Table 1. Summary of recommended therapies indicated for different types of patients.

\begin{tabular}{ll} 
Patient type & Recommended therapy \\
Out patient & No VTE prophylaxis is needed usually \\
In patient & VTE prophylaxis must be given \\
\hline Critically ill patients (ICU/ARDS) & Escalated VTE dose can be given if VTE likely to occur \\
Presumed pulmonary embolism & Therapeutic dose can be given \\
\hline Confirmed VTE event & Therapeutic VTE dose should be given \\
Post discharge & VTE prophylaxis is not recommended \\
\hline
\end{tabular}

ICU, intensive care unit; ARDS, acute respiratory distress syndrome; VTE, venous thromboembolism. 


\section{Conclusions}

With increasing cases of COVID-19 and their different presenting symptoms, physicians' approach to treating patients is under constant evolution. Hypercoagulability in COVID-19 is a relatively common finding, thus physicians should have awareness of the association between SARS-CoV-2 and its various thrombotic manifestations. However, the exact pathogenesis is unknown and further studies evaluating this finding on a bigger level are warranted. Due to the serious sequelae and poor prognosis of hypercoagulability, anticoagulation should be considered amongst SARS-CoV-2 patients. A number of studies have shown heparin to be of benefit in treating and preventing SARS-CoV-2 induced hypercoagulability, but in retrospect there have been failures in preventing coagulation in such patients despite providing prophylactic anticoagulation therapy. Studies should be conducted to identify the factors causing heparin resistance in such patients and to evaluate if the prophylactic dose needs to be raised. The cut off to switch from prophylactic dose to therapeutic dose in in-patient settings has also not been clearly defined resulting in variability in tackling coagulation among different hospitals. There is also scarce data on the effectiveness of combination therapy in treating coagulation. Further clinical and laboratory studies should be performed to discover the ideal thromboprophylaxis and management of VTE in COVID-19 patients. This will provide a standard treatment of care for such patients all over the globe.

\section{References}

1. Shi Y, Wang G, Cai X-P, et al. An overview of COVID-19. J Zhejiang Univ Sci B 2020;21:343-60.

2. Mortus JR, Manek SE, Brubaker LS, et al. Thromboelastographic results and hypercoagulability syndrome in patients with coronavirus disease 2019 who are critically ill. JAMA Netw Open 2020;3:e2011192-e.

3. Amawi H, Abu Deiab GaI, A Aljabali AA, et al. COVID-19 pandemic: an overview of epidemiology, pathogenesis, diagnostics and potential vaccines and therapeutics. Ther Deliv 2020;11:245-68.

4. Vinayagam S, Sattu K. SARS-CoV-2 and coagulation disorders in different organs. Life Sci 2020;260:118431.

5. Abou-Ismail MY, Diamond A, Kapoor S, et al. The hypercoagulable state in COVID-19: Incidence, pathophysiology, and management. Thromb Res 2020;194:101-15.

6. Campbell CM, Kahwash R. Will complement inhibition be the new target in treating COVID-19-related systemic thrombosis? Circulation 2020;141:1739-41.

7. Chen N, Zhou M, Dong X, et al. Epidemiological and clinical characteristics of 99 cases of 2019 novel coronavirus pneumonia in Wuhan, China: a descriptive study. Lancet 2020;395:507-13.

8. Huang C, Wang Y, Li X, et al. Clinical features of patients infected with 2019 novel coronavirus in Wuhan, China. Lancet 2020;395:497-506.

9. Barnes BJ, Adrover JM, Baxter-Stoltzfus A, et al. Targeting potential drivers of COVID-19: Neutrophil extracellular traps. J Exp Med 2020;217:e20200652.

10. Varga Z, Flammer AJ, Steiger P, et al. Endothelial cell infection and endotheliitis in COVID-19. Lancet 2020;395:1417-8.
11. Idell S. Coagulation, fibrinolysis, and fibrin deposition in acute lung injury. Crit Care Med 2003;31:S213-20.

12. Hanley B, Lucas SB, Youd E, et al. Autopsy in suspected COVID-19 cases. J Clin Pathol 2020;73:239-42.

13. Matos MF, Lourenço DM, Orikaza CM, et al. The role of IL6 , IL-8 and MCP-1 and their promoter polymorphisms IL-6174GC, IL-8-251AT and MCP-1-2518AG in the risk of venous thromboembolism: a case-control study. Thromb Res 2011;128:216-20.

14. Bautista-Vargas M, Bonilla-Abadía F, Cañas CA. Potential role for tissue factor in the pathogenesis of hypercoagulability associated with in COVID-19. J Thromb Thrombolysis 2020;50:479-83.

15. Cui S, Chen S, Li X, et al. Prevalence of venous thromboembolism in patients with severe novel coronavirus pneumonia. J Thromb Haemost 2020;18:1421-4.

16. Lorenzo C, Francesca B, Francesco P, et al. Acute pulmonary embolism in COVID-19 related hypercoagulability. J Thromb Thrombolysis 2020;50:223-6.

17. Zhang Y, Xiao M, Zhang S, et al. Coagulopathy and antiphospholipid antibodies in patients with Covid-19. N Engl J Med 2020;382:e38.

18. Dorgalaleh A, Dabbagh A, Tabibian S, et al. Patients with congenital bleeding disorders appear to be less severely affected by SARS-CoV-2: Is inherited hypocoagulability overcoming acquired hypercoagulability of coronavirus disease 2019 (COVID-19)? Semin Thromb Hemost 2020;46:853-5.

19. Amgalan A, Othman M. Exploring possible mechanisms for COVID 19 induced thrombocytopenia: Unanswered questions. J Thromb Haemost 2020;18:1514-6.

20. Bompard F, Monnier H, Saab I, et al. Pulmonary embolism in patients with Covid-19 pneumonia. Eur Respir J 2021;206:2932.

21. Panigada M, Bottino N, Tagliabue P, et al. Hypercoagulability of COVID 19 patients in intensive care unit. A report of thromboelastography findings and other parameters of hemostasis. J Thromb Haemos 2020;18:1738-42.

22. Guan W-J, Ni Z-Y, Hu Y, et al. Clinical characteristics of coronavirus disease 2019 in China. N Engl J Med 2020;382:170820.

23. Casey K, Iteen A, Nicolini R, Auten J. COVID-19 pneumonia with hemoptysis: acute segmental pulmonary emboli associated with novel coronavirus infection. Am J Emerg Med 2020;38:1544.e1-e3.

24. Ranucci M, Ballotta A, Di Dedda U, et al. The procoagulant pattern of patients with COVID 19 acute respiratory distress syndrome. J Thromb Haemost 2020;18:1747-51.

25. Tang N, Li D, Wang X, Sun Z. Abnormal coagulation parameters are associated with poor prognosis in patients with novel coronavirus pneumonia. J Thromb Haemost 2020;18:844-7.

26. Wichmann D, Sperhake J-P, Lütgehetmann M, et al. Autopsy findings and venous thromboembolism in patients with COVID-19: a prospective cohort study. Ann Intern Med 2020;173:268-77.

27. Lodigiani C, Iapichino G, Carenzo L, et al. Venous and arterial thromboembolic complications in COVID-19 patients admitted to an academic hospital in Milan, Italy. Thromb Res 2020;191:9-14.

28. Tang N, Bai H, Chen X, et al. Anticoagulant treatment is associated with decreased mortality in severe coronavirus disease 2019 patients with coagulopathy. J Thromb Haemost 2020;18:1094-9.

29. Emert R, Shah P, Zampella JG. COVID-19 and hypercoagula- 
bility in the outpatient setting. Thromb Res 2020;192:122-3.

30. Dane B, Smereka P, Wain R, et al. Hypercoagulability in COVID-19: Identification of arterial and venous thromboembolism in the abdomen, pelvis, and lower extremities. AJR Am J Roentgenol 2021;216:104-5.

31. Klok F, Kruip M, Van der Meer N, et al. Incidence of thrombotic complications in critically ill ICU patients with COVID19. Thromb Res 2020;191:145-7.

32.Zhou F, Yu T, Du R, et al. Clinical course and risk factors for mortality of adult inpatients with COVID-19 in Wuhan, China: a retrospective cohort study. Lancet 2020;395:1054-62.

33. Danzi GB, Loffi M, Galeazzi G, Gherbesi E. Acute pulmonary embolism and COVID-19 pneumonia: a random association? Eur Heart J 2020;41:1858.

34. Klok FA, Kruip M, Van Der Meer N, et al. Confirmation of the high cumulative incidence of thrombotic complications in critically ill ICU patients with COVID-19: an updated analysis. Thromb Res 2020;191:148-50.

35. Criel M, Jaeken J, Van Kerrebroeck M, et al. Venous thromboembolism in SARS-CoV-2 patients: only a problem in ventilated ICU patients, or is there more to it? Eur Respir J 2020;56:2001201.

36. Liu Y, Du X, Chen J, et al. Neutrophil-to-lymphocyte ratio as an independent risk factor for mortality in hospitalized patients with COVID-19. J Infect 2020;81:e6-e12.

37. Chong PY, Chui P, Ling AE, et al. Analysis of deaths during the severe acute respiratory syndrome (SARS) epidemic in Singapore: challenges in determining a SARS diagnosis. Arch Pathol Lab Med 2004;128:195-204.

38. Wong C, Lam C, Wu A, et al. Plasma inflammatory cytokines and chemokines in severe acute respiratory syndrome. Clin Exp Immunol 2004;136:95-103.

39. Connors JM, Levy JH. Thromboinflammation and the hypercoagulability of COVID 19. J Thromb Haemost 2020;18:1559-61.

40. Iba T, Thachil J. Present and future of anticoagulant therapy using antithrombin and thrombomodulin for sepsis-associated disseminated intravascular coagulation: a perspective from Japan. Int J Hematol 2016;103:253-61.

41. Opal SM. Interactions between coagulation and inflammation. Scand J Infect Dis 2003;35:545-54.

42. Duan Q, Gong Z, Song H, et al. Symptomatic venous thromboembolism is a disease related to infection and immune dysfunction. Int J Med Sci 2012;9:453.

43. Halasz G, Di Spigno F, Piepoli M, et al. [Late occurrence of pulmonary embolsim in SARS-CoV-2 pneumonia: a case series].[Article in Italian]. G Ital Cardiol (Rome) 2020;21:523-5.

44. Bangalore S, Sharma A, Slotwiner A, et al. ST-segment elevation in patients with Covid-19-a case series. N Engl J Med 2020;382:2478-80.

45. Shi S, Qin M, Shen B, et al. Association of cardiac injury with mortality in hospitalized patients with COVID-19 in Wuhan, China. JAMA Cardiol 2020;5:802-10.

46. Guo T, Fan Y, Chen M, et al. Cardiovascular implications of fatal outcomes of patients with coronavirus disease 2019 (COVID-19). JAMA Cardiol 2020;5:811-8.

47. Tavazzi G, Pellegrini C, Maurelli M, et al. Myocardial localization of coronavirus in COVID 19 cardiogenic shock. Eur J Heart Fail 2020;22:911-5.

48. Bonow RO, Fonarow GC, O'Gara PT, Yancy CW. Association of coronavirus disease 2019 (COVID-19) with myocardial injury and mortality. JAMA Cardiol 2020;5:751-3.

49. Mao L, Jin H, Wang M, et al. Neurologic manifestations of hospitalized patients with coronavirus disease 2019 in Wuhan, China. JAMA Neuro. 2020;77:683-90.

50. Ezpeleta D, Garcia D. [Manual COVID-19 para el neurólogo general].[in Spanish]. Sociedad Española de Neurología, Ediciones SEN; 2020. Available from: https:/www.sen.es/pdf/ 2020/Manual_neuroCOVID-19_SEN.pdf

51. Umapathi T, Kor AC, Venketasubramanian N, et al. Large artery ischaemic stroke in severe acute respiratory syndrome (SARS). J Neurol 2004;251:1227-31.

52. Powers WJ, Rabinstein AA, Ackerson T, et al. Guidelines for the early management of patients with acute ischemic stroke: 2019 update to the 2018 guidelines for the early management of acute ischemic stroke: a guideline for healthcare professionals from the American Heart Association/American Stroke Association. Stroke 2019;50:e344-e418.

53. Zhang Y, Cao W, Xiao M, et al. [Clinical and coagulation characteristics in 7 patients with critical COVID-2019 pneumonia and acro-ischemia].[Article in Chinese]. Zhonghua Xue Ye Xue Za Zhi 2020;41:302-7.

54. Cao Y, Liu X, Xiong L, Cai K. Imaging and clinical features of patients with 2019 novel coronavirus SARS CoV 2: A systematic review and meta analysis. J Med Virol 2020;92:1449-59.

55. Lin L, Lu L, Cao W, Li T. Hypothesis for potential pathogenesis of SARS-CoV-2 infection - a review of immune changes in patients with viral pneumonia. Emerg Microbes Infect 2020;9:727-32.

56. Li T, Lu H, Zhang W. Clinical observation and management of COVID-19 patients. Emerg Microbes Infect 2020;9:687-90.

57. Magro C, Mulvey JJ, Berlin D, et al. Complement associated microvascular injury and thrombosis in the pathogenesis of severe COVID-19 infection: a report of five cases. Transl Res 2020;220:1-13.

58. Thachil J, Tang N, Gando S, et al. ISTH interim guidance on recognition and management of coagulopathy in COVID 19. J Thromb Haemost 2020;18:1023-6.

59. Liang W, Guan W, Chen R, et al. Cancer patients in SARSCoV-2 infection: a nationwide analysis in China. Lancet Oncol 2020;21:335-7.

60. Spyropoulos AC, Ageno W, Barnathan ES. Hospital-based use of thromboprophylaxis in patients with COVID-19. Lancet 2020;395e75.

61. Paranjpe I, Fuster V, Lala A, et al. Association of treatment dose anticoagulation with in-hospital survival among hospitalized patients with COVID-19. J Am Coll Cardiol 2020;76:122-4.

62. Asakura H, Ogawa H. Potential of heparin and nafamostat combination therapy for COVID 19. J Thromb Haemost 2020;18:1521-2.

63. National Institutes of Health. Antithrombotic therapy in patients with COVID-19. 2020. available from: https://www.covid19 treatmentguidelines.nih.gov/therapies/antithrombotic-therapy/

64. Helms J, Tacquard C, Severac F, et al. High risk of thrombosis in patients with severe SARS-CoV-2 infection: a multicenter prospective cohort study. Intensive Care Med 2020;46:1089-98.

65. Miesbach W, Makris M. COVID-19: coagulopathy, risk of thrombosis, and the rationale for anticoagulation. Clin Appl Thromb Hemost 2020;26:1076029620938149.

66. Thachil J, Tang N, Gando S, et al. Type and dose of heparin in Covid 19: Reply. J Thromb Haemost 2020;18:2063-4.

67. Mummery RS, Rider CC. Characterization of the heparinbinding properties of IL-6. J Immunol 2000;165:5671-9.

68. Testa S, Paoletti O, Giorgi-Pierfranceschi M, Pan A. Switch from oral anticoagulants to parenteral heparin in SARS-CoV-2 hospitalized patients. Intern Emerg Med 2020;15:751-3. 